\begin{tabular}{|l|l|l||}
\hline \multicolumn{2}{|c|}{ PublisherInfo } \\
\hline \hline PublisherName & $:$ & BioMed Central \\
\hline \hline PublisherLocation & $:$ & London \\
\hline \hline PublisherImprintName & $:$ & BioMed Central \\
\hline \hline
\end{tabular}

\title{
Use of milrinone in non-hyperdynamic sepsis
}

\begin{tabular}{|l|l|l||}
\hline \multicolumn{2}{|c|}{ ArticleInfo } \\
\hline \hline ArticleID & $:$ & 4150 \\
\hline \hline ArticleDOI & $:$ & $10.1186 /$ ccf-1999-1362 \\
\hline \hline ArticleCitationID & $:$ & 1362 \\
\hline \hline ArticleSequenceNumber & $:$ & 9 \\
\hline \hline ArticleCategory & $:$ & Paper Report \\
\hline \hline ArticleFirstPage & $:$ & 1 \\
\hline \hline ArticleLastPage & $:$ & 3 \\
\hline \hline & & RegistrationDate : 1999-8-12 \\
\hline ArticleHistory & $:$ & OnlineDate \\
\hline \hline ArticleCopyright & $:$ & Current Science Ltd1999-8-12 \\
\hline \hline ArticleGrants & $:$ & \\
\hline \hline ArticleContext & $:$ & 1305422 \\
\hline \hline
\end{tabular}




\section{Keywords}

Cardiac output, cytokine inhibition, milrinone, non-hyperdynamic condition, sepsis, systemic inflammatory response syndrome

\section{Comments}

The use of PDEIs for specific cytokine-blocking properties is an interesting one that bears further investigation. This study used small numbers of patients in disparate groups, and not surprisingly produced no firm conclusions. Seven out of nine patients in the SIRS group had already undergone cardiac surgery and whilst cardiopulmonary bypass $(\mathrm{CPB})$ provokes a cytokine response, there are several other explanations for myocardial depression. Milrinone is experiencing an increase in popularity in weaning from $\mathrm{CPB}$. Further studies may show the beneficial effect of milronone following CPB. Also of interest is the current work on pre operative "optimisation" with PDEIs and whether an anti-inflammatory role may convey further benefit in this setting.

\section{Introduction}

Phosphodiesterase inhibitors (PDEIs) are positive inotropes and have the additional effect of inhibiting cytokine production and expression. This suggests that PDEIs may be of benefit in the treatment of patients with systemic inflammatory response syndrome (SIRS) or sepsis with a nonhyperdynamic circulatory state. This study investigated this hypothesis by comparing two groups one with SIRS or sepsis (henceforth referred to as SIRS) and a non-hyperdynamic circulatory state, and the other with congestive heart failure (CHF), to see whether milrinone conferred an additional benefit in SIRS patients.

\section{Aims}

To characterise the effect of the PDEI milrinone in adult patients with a non-hyperdynamic circulation during SIRS compared to patients with CHF. 


\section{Methods}

The study design was a prospective, protocol-driven, open labelled study conducted in an eight bed cardiology-medical ICU. Consecutive patients with SIRS (by standard criteria) were eligible for inclusion if cardiac output was less than $5.51 / \mathrm{min}$ or systemic vascular resistance (SVR) was greater than 800 dyn $X$ sec $X \mathrm{~cm}^{-5}$. These patients comprised group one. Group two was a control group of patients with CHF who required inotrope support whilst awaiting transplant or assist device implantation. Fluid resuscitation and other inotropes (dopamine/dobutamine/norepinephrine/ epinephrine) were used in addition to milrinone. Determinations of haemodynamic variables were performed every $8 \mathrm{~h}$ for at least $40 \mathrm{~h}$. Thermodilution was used for cardiac output calculations. For statistical analysis the best values after milrinone administration were compared to the average of three values prior to milrinone administration.

\section{Results}

Sixteen patients were recruited to the study (M:F 1:1). All patients had heart disease, and seven of the patients in group one were postoperative. During milrinone infusion the cardiac index increased, and SVR decreased in all patients. The SVr decrease was only significant in group two, and there was no difference in the cardiac index change between the two groups. Norepinephrine and epinephrine infusions had to be increased in the two groups, to counteract vasodilation during milrinone administration.

\section{Discussion}

Milrinone improved cardiac output to an equal extent in both groups as shown by a left ventricular stroke work index (LVSWI) improvement, despite falling pulmonary capilliary wedge pressure (PCWP). Despite the fact that PDEIs improve survival in animal models of sepsis no additional beneficial effects of milrinone were evident. It may well be that milrinone was administered too late to be of any benefit, that milrinone exerted a comparable anti-inflammatory effect in both groups, or simply that the numbers in this pilot study were too small to show a difference. Clinically, the profound vasodilating effects of milrinone may also cause a problem, even when a loading dose is not used. 


\section{References}

1. Heinz G, Geppert A, Karth JD, et al: IV Milrinone for cardiac output increase and maintenance: a comparison in non-hyperdynamic SIRS/sepsis and congestive heart failure. Intensive Care Medicine. 1999, 25: 620-624.

This PDF file was created after publication. 